\title{
Analysis of Centrality Measures of Airport Network of India
}

\author{
Manasi Sapre and Nita Parekh \\ International Institute of Information Technology, Hyderabad 500032, India \\ isanam5@gmail.com, nita@iit.ac.in
}

\begin{abstract}
In this paper we analyze the topological properties of airport network of India (ANI) using graph theoretic approach. We show that such an analysis can be useful not only in planning the infrastructure and growth of the air-traffic connectivity, but also in managing the flow of transportation during emergencies such as accidental failure of the airport, close down of the airport due to unexpected climate changes, terrorist attacks, etc. Knowledge of the connectivity pattern and load on various routes can also help in making judicious decisions for reduction of flights to contain the spread of the infectious disease.
\end{abstract}

Keywords: graph theory, centrality measures, efficiency of a network.

\section{Introduction}

In recent years, it has been observed that new influenza strains arising in one corner of the world spread rapidly affecting human lives across many countries in a very short span of time. The most recent example is that of swine flu virus, H1N1, which was first reported in April 2009 in Mexico and by August, was declared a pandemic. The main cause of the epidemic turning into pandemic in this case is the densely connected transportation services which have made the world a smaller place and the main "carriers of infectious diseases", i.e. humans can now spread the viral diseases with a much higher rate than ever before. With this view, here we analyze the connection topology of Airport Network of India (ANI) which is a subset of World Airport Network. A number of similar studies on air transportation networks have been reported both at the national level [123] and at the international level (WAN) 45]. In this study we have investigated the topological properties of ANI by representing it as a mathematical graph: each airport corresponds to a node in the network and pairs of airports connected by non-stop (direct) passenger flights are linked by edges. The role of various graph centrality measures, viz., degree, betweenness, closeness etc. to the stability of the network and the efficient flow of traffic through the whole network has been well studied [56]. Here we discuss the impact on the global efficiency of ANI by reducing connections from high-centrality nodes. Such an analysis can help in identifying nodes (airports) whose connectivity needs to be improved to increase revenue from tourism and developing more than one local hubs in different regions for efficient flow of traffic in case of undesirable situations etc. 


\section{Method}

Construction of ANI: For the construction of the ANI, data was collected for a total of 84 airports in India listed in International Civil Organization Code (ICAO) 7]. Total of 13,909 weekly direct flights from airport $i$ to $j$ from 9 major airlines have been considered (Data updated Dec, 2010) 8]. This connectivity information of flight-routes is represented by the adjacency matrix A of size $84 \times 84$, the elements of which have a value " 1 " or " 0 " depending on whether there exists an edge (i.e., connectivity) between two nodes or not. The traffic flow on the routes is incorporated by constructing a weighted ANI by assigning weights on edges proportional to the number of flights, $N_{i j}$, i.e., $w_{i j}=N_{i j} / N$, where $N$ is the total number of flights in the network. We observed that $N_{i j}=$ $N_{j i}$; i.e. the number of incoming and outgoing flights are the same. To analyze the infrastructure capacity of an airport, the strength of node $i$ is defined as $S(i)=\sum_{j=1}^{n} a_{i j} w_{i j}$ where $a_{i j}$ are the elements of the adjacency matrix and $w_{i j}$ are the weights on the edges [9].

\section{Measures Used in the Analysis of ANI:}

Efficiency: To analyze the response of the network to external factors, viz., closure of an airport, we compute global efficiency [10] as $E_{\text {glob }}(G)=1 / n(n-$ 1) $\sum_{i \neq j \in G} 1 / d_{i j}$ where $d_{i j}$ is the shortest path length between nodes $i$ and $j$.

Degree: Degree of a node $i$ is the number of nodes to which it is directly connected and is given by $k_{i}=\sum_{j=1}^{n} a_{i j}$ where $a_{i j}$ are the elements of the adjacency matrix.

Betweenness: It is defined as the ratio of number of shortest paths passing through $i$ to the total number shortest paths in the network $B_{i}=\sum_{i \neq j \neq k}$ $Z_{j-k}(i) / Z_{j-k}$ where $Z_{j-k}$ corresponds to all the shortest paths from node $j$ to node $k$ and $Z_{j-k}(i)$ corresponds to the shortest paths from node $j$ to node $k$ that pass through node $i[11]$.

Closeness: It is defined as the reciprocal of the average shortest path between a node $i$ and all other nodes reachable from it. $C l_{i}=1 / \sum_{j \in V} d_{i j}$ where $V$ is the connectivity component which contains all the vertices in the network reachable from vertex $i$. Nodes having high closeness value are most central in the network, i.e. all other nodes can be reached easily from this node.

The normalized centrality values are obtained by dividing by the maximum value such that all centrality values lie in the range 0 to 1 .

\section{Results and Discussion}

ANI Exhibits Small-world and Scale-free Properties: The clustering coefficient $(C)$ of weighted undirected ANI is $\mathbf{0 . 6 4 5}$ and its characteristic path length $(L)$ is $\mathbf{2 . 1 7}$, while the corresponding values of an equivalent randomized ANI network is 0.18 and 2.55 respectively, i.e., $C_{A N I} \gg C_{\text {rand }}$ and $L_{A N I} \sim L_{\text {rand }}$, suggesting that ANI is a small-world network [12. To analyze the distribution of flights in ANI, we considered cumulative strength distribution, $P(>S)$ as a function of strength $S$, since ANI is small network. Double Pareto law is observed 
for the distributions of the strength, $P(>S)$ as seen in Fig. 1 (a) with exponent $\gamma_{\text {cum }}^{1}=0.36$ and $\gamma_{\text {cum }}^{2}=0.71$ and for betweenness measure, $\gamma_{\text {cum }}^{1}=0.21$ and $\gamma_{\text {cum }}^{2}=0.54$ (Fig. 1 (b)). This indicates the scale-free nature of ANI, i.e., a few nodes has very large number of connections/flights while majority of nodes have very few connections. The properties of scale-free networks have been extensively studied and these networks have been shown to be robust against random removal of nodes but break down on targeted attacks 13 . Below we analyze the effect of targeted removal of high centrality nodes on the overall efficiency of the network.
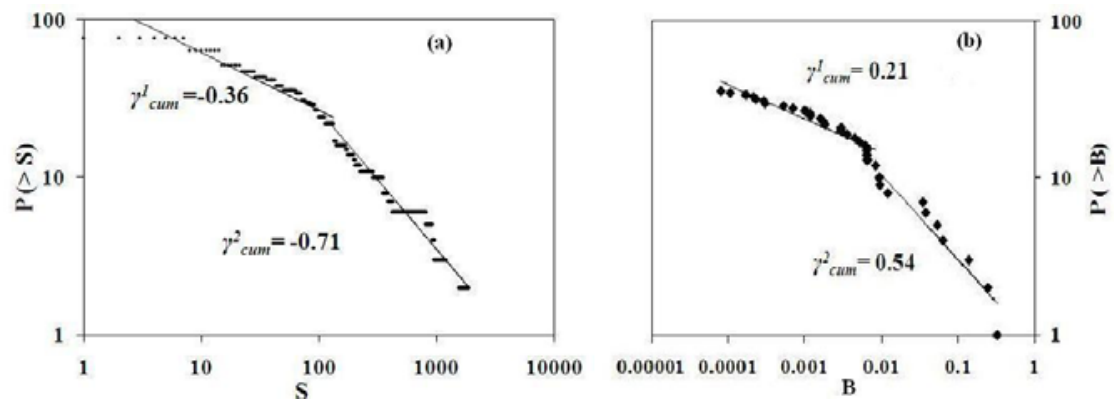

Fig. 1. (a) Cumulative strength disribution and (b) cumulative betweenness exhibit double Pareto law

The Role of High Centrality Nodes in ANI: In the event of disease spread it would be most desirous to identify crucial airports and routes to restrict transmission of disease and avoid a pandemic situation. However, complete close down of important airports or important routes is not economically viable. This led us to analyze the effect of fractional reduction of flights from an "important" airport on the overall efficiency of the network. Such an analysis would not only be useful in containing/delaying the spread of disease during an eventuality but also to assess the loss of connectivity during closure of certain airports/routes in unavoidable weather conditions, accidental failures etc. Preliminary analysis of our results has been presented in our earlier work [14.

Analysis of High Degree Nodes: In Table 1 is shown the comparison of top 10 airports listed based on their centrality values. It is clear from the table that Delhi, Mumbai and Kolkata top the list, these three being local hubs for northern, western, and eastern regions of India, respectively. The fall in connectivity in top 10 high-degree airports is $\sim 80.5 \%$, while the drop in its strength (i.e. total number of flights from it) is $\sim 89.5 \%$, clearly bringing out the effect of large number of flights on certain routes. In Fig. 2 the global efficiency of ANI is computed as a function of reduction of edges (routes) from six airports selected based on their strength. It has been shown that centrality of an airport and the socio-eonomic factors of that city are highly correlated [15. Delhi being the 
Table 1. Top 10 airports with high centrality values

\begin{tabular}{|cc|cc|cc|cc|cr|}
\hline Strength & $S_{i}$ & Degree & $k_{i}$ & Closeness & $C l_{i}$ & Betweenness $B_{i}$ & City & Cases \\
\hline New Delhi & 352 & New Delhi & 51 & New Delhi & 0.75 & New Delhi & 0.472 & New Delhi & 3703 \\
Mumbai & 314 & Mumbai & 48 & Mumbai & 0.70 & Mumbai & 0.400 & Mumbai & 3000 \\
Bengaluru & 167 & Kolkata & 33 & Kolkata & 0.63 & Kolkata & 0.220 & Chennai & 1935 \\
Kolkata & 141 & Bengaluru & 25 & Bengaluru & 0.62 & Bengaluru & 0.130 & Bengaluru & 1643 \\
Chennai & 138 & Chennai & 23 & Hyderabad & 0.58 & Chennai & 0.100 & Trichy & 1317 \\
Hyderabad & 95 & Hyderabad & 21 & Chennai & 0.57 & Hyderabad & 0.080 & Chandigarh & 1300 \\
Ahmedabad & 62 & Ahmedabad & 17 & Ahmedabad & 0.57 & Guwahati & 0.050 & Jaipur & 1008 \\
Guwahati & 53 & Goa & 13 & Goa & 0.56 & Kochi & 0.030 & Hyderabad & 773 \\
Kochi & 44 & Kochi & 11 & Guwahati & 0.55 & Ahmedabad & 0.010 & Lucknow & 673 \\
Goa & 37 & Guwahati & 10 & Kochi & 0.51 & Goa & 0.006 & Ahmedabad & 275 \\
\hline
\end{tabular}

capital and well connected to all the parts of the country, we observe that on reducing flights from Delhi has maximum effect on the global efficiency of ANI, followed by Mumbai which is the financial capital of the country. On completely removing flights from either of these two airports, the overall efficiency of the network falls by $\sim 35 \%$ as seen in Fig. 2 resulting in disconnected clusters of airports. However, in the southern part of India, the traffic flow seems to be well distributed among the three local hubs, viz., Hyderabad, Chennai and Bengaluru (sharing about 30-50\% direct flights). Removal of any one of these airports does not have any signifiant reduction in the efficiency of ANI. Though their centrality values are high, their importance in the network is reduced beause of the presence of other two local hubs in southern region which provide alternate flight-routes. Developing more than one local hub would not only ease the traffic flow but also develop healthy competition among airports resulting in improved infrastructure, reduced fares etc. as suggested by Malighetti et al [5].

The spread of infectious diseases through transportation network has become quite evident over the years. It may be noted from Table 1 that all the cities reported having high number of swine flu have either direct international flights or are directly connected to one having international flights, suggesting multiple entry points in the country. A strong correlation between cases and flights (Fig. 3), indicates the role of air transportation in the spread of disease. Thus, appropriately choosing airports/routes for reducing flights can result in reducing the impact. For example, on removing flights to Kolkata, the whole eastern region can be excluded.

Analysis of High Betweenness Nodes: From Table 1 it is clear that most high-degree nodes also have high betweenness values. It would be interesting to identify nodes having high betweenness value and low degree, e.g. Guwahati $(k=10, B=0.05)$ as it connects to remote places in eastern India. In Table 2 is summarized the effect of cutting off flights from Delhi to airports having top four ranking betweeness values. On removing flights from Delhi to Kolkata, out of 9 airports in eastern India, hop-count increases for 8 of them to reach Delhi. Similarly, on removing the Delhi-Mumbai route, 5 airports out of 18 in the western part of India are affected. Thus by restricting flights on certain routes, delay in the spread of disease can be obtained. However, no such pattern 


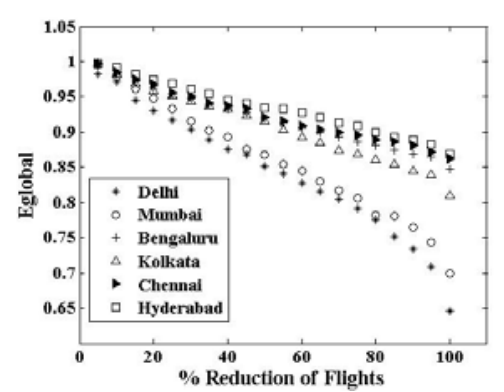

Fig. 2. The impact on $E_{\text {global }}$ of ANI as a function of reduction of flights-routes from six major hubs in ANI: Delhi, Mumbai, Bengaluru, Kolkata, Chennai and Hyderabad.

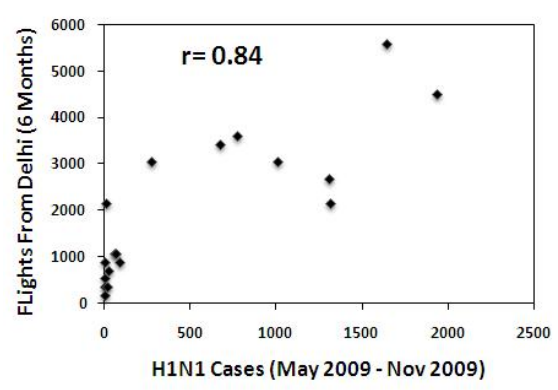

Fig. 3. Correlation between no. of flights from Delhi and no. of reported swine-flu cases (obtained from Ministry of Health of India [16]), $\mathrm{r}=0.84$

is observed in the case of removing flights from Delhi to either Hyderabad, Chennai, or Bengaluru. If similar local hubs are developed in western/northern regions, connectivity can be improved which would also help in the economic development of those regions.

Analysis of High Closeness Nodes: The closeness values indicate the accessibility of an airport to any other airport in the country. For ANI we observe that majority of airports have average closeness value $(\sim 0.45)$, suggesting a good inter-connectivity between cities. This measure can have important implication in developing tourism to hill stations (e.g., Kullu Manali, Darjeeling), wild-life sanctuaries (e.g., Corbett National Park.), historical places (e.g., Agra, Hampi) and religious places (e.g., Puri, Tirupati) apart from improving connectivity to major industrial cities (e.g., Jamshedpur). These airports, in general, do not have high-degree or high-betweenness values and in some cases are not connected by air. Their closeness values can be increased by connecting them to the nearest local hubs. Our analysis of closeness values of various tourist spots show that the most popular tourist spot, Goa (0.55), indeed has high closeness value but hill-stations, e.g., Kullu-Manali (0.37) or Agatti Island (0.33) do not, suggesting the improvement of their connectivity to improve revenue through tourism.

Table 2. The increased "hops" for airports when flights from Delhi to four highbetweenness airports are cut-off is shown.(No. given in bracket)

\begin{tabular}{|l|l|l|l|l|l|}
\hline Kolkata & $\begin{array}{l}\text { Silchar(3) } \\
\text { Lilabari(2) }\end{array}$ & $\begin{array}{l}\text { Tezpur (3) } \\
\text { Shillong(2) }\end{array}$ & $\begin{array}{l}\text { Jorhat(3) } \\
\text { Gaya(2) }\end{array}$ & Aizwal(2) & Dimapur(2) \\
\hline Mumbai & Latur(3) & Solapur(3) & Kandla(3) & Bhavnagar(2) & Nasik(2) \\
\hline Chennai & Madurai(2) & Trichy (1) & & & \\
\hline Bengaluru & Agatti(3) & Mangaluru(2) & & & \\
\hline
\end{tabular}




\section{Conclusion}

Graph theoretic analysis of weighted ANI helps in identifying "critical" nodes, not necessarily the ones with high connections but also the ones lying on high traffic-routes (high betweenness) or geographically well distant nodes (high closeness). Analysis of these high-centrality nodes can help in improving efficiency of ANI, tourism in the country and containing the spread of disease.

\section{References}

1. Li, W., Chai, X.: Statistical analysis of airport network of China. Phys. Rev.E. 69, 46106 (2004)

2. Guida, M., Funaro, M.: Topology of Italian airport network. Chaos Solitons and Fractals 31, 527-536 (2007)

3. Bagler, G.: Analysis of airport network of India as a complex weighted network. Physica A 387, 2972-2980 (2008)

4. Guimera, R., Mossa, S., Turtschi, A., Amaral, L.A.N.: The worldwide air transportation network: Anomalous centrality, community structure and cities' global roles. PNAS 2, 7794-7799 (2005)

5. Malighetti, G., Martini, G., Paleari, S., Redondi, R.: The Impacts of Airport Centrality in the EU Network and Inter-Airport Competition on Airport Efficiency. MPRA (2009)

6. Berger, A., Müller-Hannemann, M., Rechner, S., Zock, A.: Efficient computation of time-dependent centralities in air transportation networks. In: Katoh, N., Kumar, A. (eds.) WALCOM 2011. LNCS, vol. 6552, pp. 77-88. Springer, Heidelberg (2011)

7. http://www.icao.int

8. The data from the sites of major airlines (2010)

9. Barrat, A., Barthélemy, M., Pastor-Satorras, R., Vespignani, A.: The architecture of complex weighted network. Proc. Natl. Acad. Sci (USA) 101(11), 3747-3752 (2004)

10. Latora, V., Marchiori, M.: Efficient Behavior of Small World Networks. Phys. Rev. Lett. 87, 198701 (2001)

11. Newman, M.E.J.: The structure of scientific collaboration network. Proc. Natl. Aca. Sci. 98, 404-409 (2001)

12. Watts, D.J., Strogatz, S.H.: Collective dynamics of 'small-world' networks. Nature 393, 440-442 (1998)

13. Barabási, A.-L., Albert, R.: Emergence of scaling in random networks. Science 286, 509-512 (1999)

14. Sapre, M., Parekh, N.: Analysis of Airport Network of India. In: Poster presentation at Grace Hopper Coneference in Computer Science, Bangalore (2010)

15. Wang, J., Mo, H., Wang, F., Jin, F.: Exploring the network struture and nodal centrality of China's air transport network: A complex network approach. Journal of Transport Geography (in press) (2010)

16. http://www.mohfw.nic.in/ 